\title{
Humano, demasiado humano, ultrahumano El desafío de Nietzsche al humanismo ${ }^{1}$
}

\author{
Human, All too Human, Transhuman \\ Nietzsche's Challenge to Humanism ${ }^{2}$
}

Helmut Heit ${ }^{3}$

Kolleg Friedrich Nietzsche. Klassik Stiftung Weimar, Alemania

Recepción: 20 de junio del 2020

Evaluación: 03 de julio del 2020

Aceptación: 20 de julio del 2020

1 Este artículo es un resultado del proyecto de investigación "Nietzsche's Philosophy of Modernity", que fue auspiciado por la Tongji Universität Shanghai, de la República Popular de China, en el marco del "Double First Class Development Project of Tongji University". Una parte de esta contribución fue publicada originalmente en alemán en Heit, 2018.

2 Traducción del alemán por Mikhail Krasnov, Instituto Internacional de Idiomas, Universidad Pedagógica y Tecnológica de Colombia. Revisión conceptual, corrección y complementos a la traducción por Alfredo Rocha de la Torre, Escuela de Filosofía, Universidad Pedagógica y Tecnológica de Colombia.

3 Dr. Phil. por la Leibniz Universität Hannover (Alemania), Profesor asociado en la Tongji University Shanghai y Director del Kolleg Friedrich Nietzsche der Klassik Stiftung Weimar.

Correo electrónico: helmut.heit@klassik-stiftung.de

Heit, H. (2020). Humano, demasiado humano, ultrahumano. El desafío de Nietzsche 


\title{
Resumen
}

Nietzsche se burla del humanismo en reiteradas ocasiones, en parte debido a su antropología naturalizada, no obstante el hecho de que los conceptos de superación de sí mismo, de refinamiento y liberación son presentados regularmente en sus escritos. Este artículo señala que el nexo entre el naturalismo y el perfeccionismo en el pensamiento de Nietzsche deriva del 'enigmático doble-carácter' de la naturaleza humana y culmina en una antropología política de la libertad. La primera sección explica el discurso de Zaratustra acerca del superhombre, no como una doctrina o un mandamiento, sino como un intento de persuasión y una invitación a alcanzar el desarrollo superior de la humanidad. La segunda sección muestra la forma como Nietzsche utiliza sus lecturas de fisiología de su época para elaborar una concepción de lo humano en términos de una dinámica de organización corpórea. En la tercera sección se muestra que el desarrollo natural e histórico-cultural de la especie humana ha creado una posibilidad para impulsar conscientemente una transformación. El proyecto de una disposición plausible estéticamente no está determinado natural ni moralmente, sino que resulta de una-como yo la denomino- 'fuerza normativa de lo posible', que puede ser realizada a través de un ejercicio cultural. El reto de Nietzsche al humanismo es una invitación a ser mejores seres humanos.

Palabras clave: Libertad, superación de sí mismo, naturalismo, perfeccionismo, humanismo, transhumanismo.

\begin{abstract}
Nietzsche mocks humanism at various occasions, in part because of his naturalized anthropology, while concepts of human self-overcoming, refinement, and liberation are regularly present in his writings. This paper argues that the connection between naturalist and perfectionist aspects in Nietzsche's thought derive from the 'mysterious double-character' of human nature and result in a political anthropology of freedom. The first section explains Zarathustra's talk of the Over-Human not as a doctrine or commandment, but as persuasion and invitation to attempt a higher development of humanity. The second section shows how Nietzsche uses his readings of contemporary physiology to develop a conception of the human as a dynamic body-organization. In the third section, it is argued that the history of nature and culture of the human species has created a possibility for further conscious transformation. The
\end{abstract}


ambition to design an aesthetically approvable character is neither naturally determinated nor morally required, but results from a - as I call it - 'normative force of the possible', which can be realized through cultural exercise. Nietzsche's challenge to humanism is an invitation to become better humans.

Keywords: Freedom, self-overcoming, naturalism, perfectionism, humanism, transhumanism. 
El hombre en sus más nobles y elevadas funciones es enteramente naturaleza y porta en sí mismo su inquietante doble carácter (Nietzsche, KSA 1, p. 783).

\section{¿Nietzsche como enemigo del humanismo?}

Nietzsche parece tener poco que ver, en general, con el humanismo, especialmente cuando este término está marcado por el artículo definido como una doctrina filosófica. Sus declaraciones explícitas acerca del término suelen ser, especialmente a primera vista, negativas y de rechazo. Se podría pensar que sus reflexiones sobre este tema culminan en la triple exclamación de Más allá del bien y del mal: "¡Oh Voltaire! ¡Oh humanitarismo! ¡Oh imbecilidad!" (Nietzsche, 2013, p. 77; KSA 5, p. 54)4. De hecho, más recientemente, Volker Gerhardt, entre otros, tomó esta observación como una oportunidad para distinguir su propio concepto de humanidad de los reproches y objeciones de Nietzsche. Según Gerhardt, uno debe defender la idea de amar en cada hombre individual al mismo tiempo la humanidad, contra un "desdén común (...) a cuya cabeza lamento encontrar a Friedrich Nietzsche con su inmaduro desprecio por la palabra humanidad" (Gerhardt, 2019, p. 37). Por supuesto, al comprobado experto en la filosofía de Nietzsche no se le escapa el hecho de que éste, en su programática del individuo productivo y soberano, puede estar "más cerca de la idea de humanidad que muchos otros pensadores del siglo XIX" (Gerhardt, 2019, p. 37). Gerhardt tampoco deja ninguna duda de que una filosofía humanista debe reconocer en primer lugar que el hombre nace, es completamente natural y sigue siendo parte inseparable del contexto natural. Todas las más altas capacidades del hombre no están solamente ligadas al origen, sino de forma permanente "ligadas a la presencia del cuerpo", como aclara Gerhardt con vistas al discurso de Zaratustra acerca de la gran razón del cuerpo (Gerhardt, 2019, p. 96). Incluso la idea del superhombre, que parece estar en contradicción directa con las concepciones del humanismo clásico que apuntan a la universalidad y la igualdad, puede entenderse como el ideal de un ser humano superior, en el que "una intención humanista tiene un efecto" (Gerhardt, 2019, p. 39). Si bien algunos aspectos de la filosofía de Nietzsche pueden ser consistentes con el concepto de humanidad de Gerhardt, probablemente la diferencia decisiva resulta del diseño

\footnotetext{
${ }^{4}$ Las traducciones de los textos de Nietzsche al español, realizadas por Andrés Sánchez Pascual, son por lo general las versiones utilizadas en este artículo. En los casos en que no sea referida la correspondiente versión castellana, se sobreentiende que la versión pertenece al traductor de este escrito.
} 
universalista que este autor ilustra en la distinción entre filantropía y humanidad: "Mientras que la filantropía está relacionada con un comportamiento de apertura, de ayuda y de preocupación ante el otro existente $-\mathrm{y}$ con ello está vinculada gustosamente con modos concretos de comportarse-, presupone a la humanidad (Menschheit) en su generalidad en el hombre particular (Gerhardt, 2019, p. 32).

Es precisamente esta orientación, que tiene su origen en Kant, según la cual una ética y política humanista es efectiva o concebible si las razones dadas para ello "derivan su poder de persuasión de su validez general" (Gerhardt, 2019, p. 225), la que no llega a ser compartida por Nietzsche. Las diferencias entre Nietzsche y Gerhardt a este respecto parecen claras, y quizás Gerhardt también haga bien en contornear de esta manera su propia posición contra el papel de la crítica en Nietzsche. Sin embargo, vale la pena, en mi opinión, considerar la contribución de Nietzsche a una filosofía de lo humano, y también a una filosofía humana, de una manera diferente. Como punto de partida me gustaría hacer una cita que antepongo también en este ensayo como un lema. Al comienzo de la introducción al quinto de sus libros no escritos para Cósima Wagner, El duelo en Homero, se encuentran ya los motivos centrales de sus reservas contra el humanismo tradicional:

Cuando se habla de humanidad se piensa en lo que separa y distingue al hombre de la naturaleza. Pero tal separación no existe en realidad: las propiedades 'naturales' y las denominadas propiamente 'humanas' son inseparables. El hombre en sus más nobles y elevadas funciones es enteramente naturaleza y porta en sí mismo su inquietante doble carácter. Sus terribles capacidades, consideradas como inhumanas, son incluso quizás el terreno más fructífero en el que pueden crecer todos aquellos impulsos, hechos y obras de toda humanidad (Nietzsche, KSA 1, 783).

En estas tempranas reflexiones escritas en los días navideños del 1872, y aun bajo el claro hechizo de su amor por Wagner y Schopenhauer, se encuentran ya los motivos centrales de su desarrollo posterior, al menos en relación con la pregunta central para el tema del humanismo; la pregunta por la antropología filosófica. En una clara posición negativa al respecto, Nietzsche se opone a una concepción idealista de que el hombre deba distinguirse esencialmente de la naturaleza y que nuestras cualidades y logros culturales, que en general se consideran especialmente valiosos, deban explicarse por este carácter sobre o extranatural del hombre. Por el contrario, desde temprano Nietzsche insiste en que las llamadas capacidades naturales, y las llamadas capacidades 
culturales del hombre, están "entretejidas inseparablemente", es decir, representan una continuidad. A diferencia de muchos pensadores anteriores, Nietzsche no define al hombre como un zoon logon echon y como un animal rationale, pues en general ya no pertenece a aquellos que ven en la razón la cualidad más distinguida y distintiva del hombre. En lugar de distinguir a los humanos de otros animales sobre la base de su racionalidad y, en general, de distinguir la razón de la naturaleza, Nietzsche quiere "(...) retraducir el hombre a la naturaleza" (2013, p. 223; KSA 5, p. 169). Es por esto que Nietzsche ha sido puesto una y otra vez, y no sin razón, en relación con antropologías naturalistas. Por otro lado, los pensamientos e imágenes de la superación de sí mismo y del supremo desarrollo, del refinamiento, de la liberación y trascendencia están siempre presentes en sus escritos (Heit, 2018).

Con esta naturalización del hombre se vincula, ya en El duelo en Homero, la conjetura teórica cultural de Nietzsche, según la cual las instituciones y logros con los cuales nos distinguimos en tanto humanos de la otra naturaleza, y también nos elevamos sobre ella, son a su vez producto de fuerzas y acciones que hoy son consideradas inhumanas. Esta sospecha consiste en que el llamado 'malvado' no solo no es una simple contradicción del denominado 'bueno', sino que, tanto históricamente como en la actualidad, posiblemente el uno tiene al otro como condición. La metáfora en la que estos elementos conflictivos del hombre se unen es el discurso acerca del "inquietante doble carácter". Deseo seguir esta expresión, relacionándola con otro pasaje igualmente enigmático, aunque mucho más conocido, como lo es el del prólogo de Así habló Zaratustra:

Habéis recorrido el camino que lleva desde el gusano hasta el hombre, y muchas cosas entre vosotros continúan siendo gusano. En otro tiempo fuisteis monos, y aun ahora es el hombre más mono que cualquier mono. Y el más sabio de vosotros es tan solo un ser escindido, híbrido de planta y fantasma. Pero ¿os mando yo que os convirtáis en fantasmas o en plantas? ¡Mirad, yo os enseño el superhombre! El superhombre es el sentido de la tierra. Diga vuestra voluntad: ¡Sea el superhombre el sentido de la tierra! (1993, p. 34; KSA 4, p. 14).

Sobre la base de una interpretación ejemplar de este pasaje, no solo se pueden concretar los problemas, sino también las posibilidades conceptuales de un humanismo filosófico. En las primeras líneas de esta cita, Zaratustra lleva a cabo una narrativa con fuertes ecos biológico-evolutivos. Los hombres interpelados en el colectivo son puestos en una conexión diacrónica y sincrónica 
con el gusano y, más claramente, en asociación con el darwinismo, con el mono. Sin duda, el hombre ha recorrido un largo camino desde el gusano y el mono hasta su estado actual, pero es al mismo tiempo en muchos aspectos un reptil y ridículamente irracional. El hombre se caracteriza entonces por una simultaneidad tensa y disonante. Incluso una persona sutil, educada y cultivada, es al mismo tiempo solo la antítesis entrelazada (¿inextricablemente?) de la mera espiritualidad y de la naturaleza espiritualmente viva. La referencia a la planta y al fantasma lleva al dualismo clásico a una fórmula irónica y, al mismo tiempo, lo trasciende en una figura híbrida ambigua, especialmente porque uno sabe bien que no existen fantasmas. De esta manera se pueden entender los comentarios de Zaratustra en el contexto del discurso naturalista contemporáneo, en el que Gerhardt también inscribe la naturaleza del hombre. Al mismo tiempo, la aliteración metafórica de ambigüedad e hibridación enfatiza también, sin embargo, una unidad de opuestos y, por tanto, una tensión dinámica, una carga y una fuerza de la cual surgen nuevas opciones.

Con la posterior pregunta retórica, el texto deja lo meramente diagnóstico y va en dirección del futuro desarrollo. Sin embargo, Zaratustra no cambia repentinamente de lo doctrinal a lo normativo e imperativo, sino más bien a lo protréptico y a lo persuasivo. La tensión dinámica debe hacerse fructífera y ser dirigida en cierta dirección. El narrador llama a evitar la alternativa falsa del fantasma y la planta, la mente y la naturaleza y, en cambio, anuncia de manera no individualista otra idea a un colectivo: "Os enseño el superhombre" y "El superhombre es el sentido de la tierra" (Nietzsche, 1993, p. 34; KSA 4, p. 14). Si en el contexto de la narrativa del Zaratustra este pasaje nos permite hablar del superhombre como una doctrina de Nietzsche, es algo que puede permanecer sin ser decidido por el momento. De interés en el contexto de mis elaboraciones está más bien la conexión retórica de una narrativa evolutiva con una llamativa invitación, que trasciende las alternativas actuales con la ayuda de una nueva metáfora. La afirmación solo supuestamente constatable de que el superhombre "es" el sentido de la tierra, pertenece a este contexto retórico. El carácter persuasivo de esta constatación (Fest-Stellung) surge en la siguiente aclaración: "Vuestra voluntad dirá: ¡el superhombre sea el sentido de la tierra!" (Nietzsche, 1993, p. 34; KSA 4, p. 4). Esta frase no expresa doctrina alguna, sino una invitación. La supuesta voluntad libre de un destinatario en plural debe ser motivada hacia una determinada decisión, a saber, a definir al superhombre como sentido global. Esta definición del sentido no se refiere en primer lugar a una existencia individual, sino a la totalidad del mundo humano. En segunda instancia, el subjuntivo enfatiza el contrafactual trascendente de esta disposición. La palabra superhombre no se 
refiere a un ideal moral, sino a una atractiva posibilidad. Es una invitación a la autosuperación de la especie humana.

Ciertamente, la interpretación de un solo pasaje, especialmente de Zaratustra, difícilmente puede ser considerada como una reconstrucción general de la antropología filosófica de Nietzsche, y de hecho no se entiende como tal. Este pasaje ejemplifica más bien una conexión original de alternativas supuestamente exclusivas. Se podría con ello superar las dicotomías clásicas de naturalismo versus trascendencia, y de existencialismo individualista versus antropología sociológica cultural. A continuación me gustaría señalar que Nietzsche esboza una propuesta para la autocomprensión filosófica del hombre, que vincula motivos naturalistas con una praxis del trascender. Sus procedimientos de naturalización son tan específicamente suyos que la etiqueta 'naturalismo' tiende a causar más confusión que lo que aporta en claridad. Además, no solo se centra en existencias aisladas, sino que también toma en consideración la naturaleza fundamentalmente social de lo humano. Lo social se manifiesta no solo en las altas instituciones histórico-culturales del hombre, principalmente en el lenguaje, sino también en la naturaleza humana en tanto organización viva y jerárquica. La individualización es el producto de la organización social, y es la que permite al sujeto desarrollar la capacidad de autoformación y autodeterminación. Nietzsche demuestra ser un filósofo filantrópico y un defensor de una antropología política de la libertad. Sus objeciones al humanismo clásico son como las críticas productivas que se pueden esperar de un buen amigo.

\section{El hombre como animal demasiado humano}

La dimensión naturalista de la concepción nietzscheana del hombre ha sido resaltada regularmente en la investigación ${ }^{5}$. Sin embargo, el hecho de que él asocie repetidamente la naturalización con la terminología sociopolítica ha recibido menos atención hasta ahora ${ }^{6}$. Un punto de partida común para sus consideraciones a este respecto es el cuarto discurso de Zaratustra, ya referido por Volker Gerhardt. Dirigida a los despreciadores del cuerpo, la distinción entre cuerpo (Leib) y alma en el sentido de dos existencias separadas

\footnotetext{
5 Realizo un resumen de las interpretaciones naturalistas de Nietzsche y sus dificultades en Heit (2016a). En Heit (2013), discuto más detalladamente en qué sentido Nietzsche considera al sujeto humano como parte de la naturaleza.

6 Una excepción, aparte de Jyung-Hyun Kim (1995), es Herman Siemens (2016).
} 
es caracterizada como infantil. Sin embargo, el discurso del niño no es rechazado como completamente inútil, pues "¿por qué no se debe hablar como los niños?” (Nietzsche, KSA 4, p. 39). La concepción dualista no es refutada o generalmente prohibida, sino desacreditada en ciertos contextos. Parece inmadura e ingenua, irreflexiva y mal informada, hace hincapié en lo increíble y sucumbe a la seducción del lenguaje. En el contexto de las investigaciones filosóficas, el dualismo ya es admisible. La visión alternativa, por otro lado, establece una unidad psicofísica: "Pero el despierto, el sapiente, dice: cuerpo soy yo íntegramente, y ninguna otra cosa; y alma es sólo una palabra para designar algo en el cuerpo" (Nietzsche, 1993, p. 60; KSA 4, p. 39). El alma es subsumida en el cuerpo. Sin embargo, este naturalismo aparentemente monista no se presenta como una verdad científica comprobada, sino como un estado de alerta y conocimiento equipado con los atributos de una mentalidad intelectualmente superior. Solo por esta razón es aconsejable comprender este motivo naturalista en el sentido de una heurística interesada por la ciencia y no como una doctrina basada en la ciencia ${ }^{7}$.

En una nota muy informativa del contexto de su trabajo preparatorio sobre el Zaratustra, Nietzsche aplica explícitamente la reserva epistemológica a un ideal científico de interpretación de las condiciones de existencia del sujeto: "Para que exista un sujeto, debe haber aquí algo que persista" (Nietzsche, Nachlass 1881 11[268], KSA 9, p. 543). Tanto la existencia como la percepción autoconsciente de la subjetividad requieren una cierta estabilidad, un permanecer-se idéntico en el espacio y en el tiempo. Pero esta persistencia, que después de hablar de identidad personal es idéntica incluso a sí misma, se echa de menos bajo el presupuesto del cambio y la transformación permanentes. Nietzsche no atribuye, por tanto, la posibilidad de subjetividad a una existencia "propia" o "auténtica" de lo que persiste, sino a un productivo engaño:

Sin embargo, creo ahora: el sujeto podría surgir en tanto surge el error de lo idéntico (Gleichen), por ejemplo cuando un protoplasma de fuerzas diversas (la luz presiona la electricidad) recibe siempre solamente un impulso particular y de acuerdo con este impulso colige una identidad (Gleichheit) de las causas: o cuando en general solamente se está en capacidad de recibir un impulso y todos los otros son sentidos como idénticos (Nachlass 1881 11[268], KSA 9, pp. 544s.).

7 Schacht (2012) destaca especialmente esta diferencia. 
Es de destacar que Nietzsche refiere de manera casual y ejemplar, precisamente, a las tres fuerzas a las que también se dirige Hermann von Helmholtz para explicar los procesos de percepción óptica. Independientemente de si la retina se estimula visual, eléctrica o mecánicamente, nuestro organismo 'interpretará' siempre inevitablemente este estímulo como luz. Por esta razón, entre otras cosas, Helmholtz se ve a sí mismo autorizado para hablar de la percepción como una operación activa de los órganos sensoriales, que llegan a juzgar el mundo exterior con la ayuda de "conclusiones inconscientes de las sensaciones sobre sus causas", con lo cual su validez, como en todas las conclusiones de inducción y analogía, está infradeterminada (Helmholtz, 1867, p. 430; Schiemann, 2014, pp. 46-75). Al mismo tiempo, lo simple y lo persistente son indispensables para la educación y la preservación de la vida y la subjetividad. Por esta razón cuando Nietzsche afirma que "(...) la simplificación es la necesidad fundamental de lo orgánico" (Nachlass 1881 11[315], KSA 9, p. 563), se relaciona de esta manera con la fisiología contemporánea. Un organismo vivo no solo se basa en estos supuestos, sino que los realiza también en cierta medida mientras dure su existencia; ellos son efectivos y reales en él y a través de él. De hecho, en este sentido, el "error es el padre de lo vivo" (Nietzsche, Nachlass 1881 11[270], KSA 9, p. 545).

De particular interés en el campo de la antropología filosófica, por lo tanto, aparte de la reserva cognitiva crítica, es ante todo que los engaños y las simplificaciones son funcionales y se convierten en una realidad viva en el organismo. En este sentido también podemos entender la continuación del discurso que Zaratustra pone en boca del despierto y del sapiente: "El cuerpo es una gran razón, una pluralidad dotada de un único sentido, una guerra y una paz, un rebaño y un pastor" (1993, p. 60; KSA 4, p. 39). Este denso enunciado no solo afirma cuatro veces lo que es el cuerpo, sino que simultáneamente plantea una serie de preguntas. Se prestó especial atención con ello a la primera determinación del cuerpo como una gran razón (Gerhardt, 2011). A través de esta memorable fórmula se atribuye la facultad del espíritu, hasta ahora más distinguida, al cuerpo o se identifica con él. Esta es una de las razones por las cuales se demostró que Nietzsche fue pionero en las nuevas discusiones acerca de la encarnación (Embodiment) y la cognición encarnada (embodied cognition). Sin embargo, las relaciones entre el cuerpo, el yo y el sí-mismo, y entre la gran y la pequeña razón, siguen siendo algo enigmáticas. Me parece útil en este contexto prestar atención a las otras tres determinaciones, que giran en torno a la relación entre la unidad y la multiplicidad. El cuerpo en sí mismo no es monista, sino una pluralidad caracterizada por un sentido 
común y por metáforas del conflicto interno y de la tensa organización. El cuerpo consiste en la simultaneidad de multiplicidad disonante y unanimidad, conflicto y armonía, séquito y enseñoreamiento. El hombre vivo puede entenderse como una "organización corporal” dinámica-agonal (Abel, 2001, p. 31).

Las metáforas del pastor y del rebaño, especialmente, en las que se combinan connotaciones biológico-naturalistas con connotaciones políticas y sociales, pueden dilucidar decisivamente la relación entre libertad y determinismo, así como entre el individuo y la sociedad. Sin embargo, con una pregunta tan sistemática se sale adelante casi sin referencia al escrito póstumo. Las principales características de una "socio-fisiología del yo" pueden observarse ante todo en las notas de principios de la década de 1880, en las que Nietzsche, según la observación de Herman Siemens, "profundiza su giro hacia la fisiología" y desarrolla "una prehistoria socio-fisiológica del individuo" (Siemens, 2016, p. 167), no obstante que la idea fundamental ya se encuentra en El duelo en Homero. En La gaya ciencia (1882) y en Así habló Zaratustra (1883/85) ya juegan estas características un claro, aunque subterráneo rol, sin ser precisadas detalladamente. Esto está reservado para los escritos posteriores al Zaratustra, que están fuertemente influenciados por las lecturas y reflexiones fisiológicas. En una formulación más contundente del verano de 1885, como es típico de los escritos póstumos, se unen en el fondo todas las reflexiones para la comprensión de la antropología fisio-política de Nietzsche. El apunte está en el contexto de reflexiones críticas al cogito cartesiano, que se han incorporado reformuladas al primer capítulo de Más allá del bien y del mal. Aunque solo es un apunte no publicado por Nietzsche, el texto contribuye significativamente a la comprensión temática de su presunta antropología y de su obra publicada en vida.

En este apunte Nietzsche pregunta: "Punto de partida del cuerpo y de la fisiología: ¿por qué razón?”. Inmediatamente incluye la respuesta: “-Logramos la correcta representación del talante de nuestra unidad-subjetiva (...)" (Nachlass 1885 40[21], KSA 11, p. 638). Al caracterizar expresamente la fisiología como un "punto de partida" del cual podemos derivar la "correcta representación", él subraya la función hipotética de ésta. Nietzsche no desarrolla su antropología como un científico natural, sino (solo) en relación al "hilo conductor del cuerpo" (Nachlass 1885 36[35], KSA 11, p. 565), como se afirma repetidamente en otras partes del escrito póstumo (Sommer, 2016, p. 152). A diferencia de las más sutiles capacidades de la razón, Nietzsche 
considera que el cuerpo es capaz de ofrecer un testimonio de carácter informativo. La fisiología, y con ello el naturalismo y el sensualismo, son convenientes como "hipótesis reguladoras" o como "principios heurísticos" debido a su productiva función epistemológica (Nietzsche, 2013, p. 43; KSA 5, p. 29). Partiendo de esta perspectiva, se logra una concepción más confiable de la unidad del sujeto "(...) como regente en la cima de la comunidad, no como 'almas' o 'fuerzas vitales', así como también de la dependencia de estos regentes ante los gobernados, y de las condiciones de jerarquía y división del trabajo como posibilitación simultánea del individuo y del todo" (Nieztsche, Nachlass 1885 40[21], KSA 11, p. 638). La atención de Nietzsche se centra en la naturaleza específica de la constitución del sujeto, cuya existencia no se niega. Su demarcación se refiere en particular a la negación de las especulaciones platónico-cristianas-cartesianas acerca del alma, así como al vitalismo contemporáneo $^{8}$ (Ioan, 2015, p. 219). El sujeto es una organización jerárquica, política y funcional de la división del trabajo.

Nietzsche precisa estas determinaciones en las siguientes líneas. En primer lugar, las "unidades vivas" se caracterizan por su carácter emergente y por su transitoriedad, ya que "la eternidad no pertenece al "sujeto" (Nietzsche, Nachlass 1885 40[21], KSA 11, p. 638); el sujeto es enteramente mortal. En segundo lugar, se muestra en el sujeto una lucha que se expresa en obediencia y mando, ya que "a la vida pertenece una fluyente delimitación del poder" (Nietzsche, Nachlass 1885 40[21], KSA 11, p. 638). Con esta determinación se muestra, especialmente, la dimensión política que Nietzsche considera, una y otra vez, como subyacente a su concepción de sujeto. En Más allá del bien y del mal Nietzsche traduce la pregunta acerca de 'quién se es' en la pregunta "en qué orden jerárquico se encuentran recíprocamente situados los instintos (Triebe) más íntimos de su naturaleza" (2013, p. 35; KSA 5, p. 20). Unas páginas más adelante, Nietzsche hace referencia al "alma como estructura social de instintos (Triebe) y afectos" (2013, p. 43; KSA 5, p. 27), y luego al cuerpo como una "estructura social de muchas almas" (2013, pp. 51-52; KSA 5, p. 33). El apunte del escrito póstumo especifica dos aspectos adicionales que pueden entenderse con la ayuda de metáforas políticas. En tercer lugar, una "cierta ignorancia en la que el gobernante se mantiene por

\footnotetext{
8 Razvan Ioan ha demostrado convincentemente que Schopenhauer es un padrino y un objeto de crítica, ya que Nietzsche no solo se distingue (con Schopenhauer) del alma clásica, sino también de su idea de la fuerza vital.
} 
encima de los asuntos individuales e incluso de los disturbios de la comunidad", pertenece no solo a las condiciones funcionales necesarias del organismo, sino también a su capacidad de control (Nachlass 1885 40[21], KSA 11, p. 638). A partir de la comprensión de la ignorancia funcional del regente, se produce además "una valoración para el no saber, que visto en líneas generales es el simplificar y falsear, lo perspectivista" (Nachlass 1885 40[21], KSA 11, pp. 638s.), que al final del apunte Nietzsche reafirma a favor de su heurística fisiológica en lugar de la introspección y la reflexión: "El cuestionamiento directo del sujeto sobre el sujeto, y toda autorreflexión del espíritu, tiene sus peligros en que podría ser útil e importante para su actividad de malinterpretarse" (Nachlass 1885 40[21], KSA 11, p. 639). Si bien esta dimensión heurística vuelve a ser relevante al final de mi reflexión, en este punto me interesa principalmente una cuarta precisión, que Nietzsche enfatiza en particular: "Lo más importante es, sin embargo: que vemos al dominador y a sus dominados de la misma manera, todos sintiendo, queriendo, pensando (...)" (Nachlass 1885 40[21], KSA 11, p. 639).

A la luz de estas líneas pueden entenderse de mejor manera las peculiaridades específicas de la combinación de conceptos naturalistas y políticos en la antropología de Nietzsche. Los regentes y los gobernados son del mismo tipo; las diferencias de rango entre ellos son graduales y no son fundamentales. También se deduce que la jerarquía es variable. Por lo tanto, ésta no tiene que basarse, como lo supone Gerhardt, en el concepto de igualdad como criterio ${ }^{9}$ (Gerhardt, 2019, p. 38). La jerarquía tampoco representa un orden 'correcto, 'ideal' o 'natural' más o menos bueno, sino que es precisamente tal como ella es. En una unidad viva se forman coaliciones más o menos estables sobre la base de procedimientos de negociación agonistas, en los que la fuerza superior, así como el reconocimiento voluntario debido a objetivos comunes o superpuestos, pueden cumplir un rol. Todas las partes de esta unidad existen sintiendo (epitimótico), queriendo (timótico) y pensando (lógico). Solo por esta razón no es útil asociar la concepción del alma de Nietzsche con las tres partes cualitativamente distintas del alma de la politeia platónica, como ha sucedido ocasionalmente. Sin embargo, en el contexto del discurso de "la estructura social de los instintos y los afectos" (Nietzsche, 2013, p. 43; KSA 5, p. 27), se afirma que "debe estar claro que Nietzsche toma su perspectiva homuncular de los instintos del tratamiento platónico de las

9 "La igualdad es en esto la medida que permite medir el rango de la grandeza humana".

Heit, H. (2020). Humano, demasiado humano, ultrahumano. El desafío de Nietzsche 
partes del alma" (Clark and Dudrick, 2015, p. 272). Esta especulación está basada probablemente en la plausibilidad general de la referencia siempre correcta de Nietzsche a Platón, pero al mismo tiempo ignora la literatura fisiológica y psicológica realmente consultada por Nietzsche. De esta manera, se puede observar un paralelo en el hecho de que Nietzsche también entiende al sujeto como una composición de partes normativamente densas, aunque las diferencias predominan tanto que el intento de construir la propuesta de Nietzsche como un "refinamiento" de la teoría de Platón (Clark and Dudrick, 2012, p. 167) finalmente conduce a demasiados desvíos.

En primera instancia debe resaltarse que Nietzsche, en contraste directo y explícito con Platón, asume la mortalidad del alma (2013, p. 38; KSA 5, p. 23; Janaway, 2014, p. 14). Es por esto que no está claro si Platón realmente califica como un crítico del atomismo del alma, como tampoco si Nietzsche lo percibió como tal. Evidentemente el "relato de John Cooper acerca de la psicología de Platón" (Clark and Dudrick, 2012, p. 164) no contribuye en nada a la comprensión de la interpretación de Nietzsche acerca del filósofo griego, debido a que Nietzsche no conoció ni podría haber conocido obviamente la lectura de Cooper de 1998. Por el contrario, la historia de la religión europea de Julius Lippert se encuentra aún hoy en día con algunos rastros de lectura en la biblioteca personal de Nietzsche.

En el capítulo acerca de la fisiología platónica dice Lippert: “Aunque en las mentes se asienta el alma divina e inmortal con sus 'vuelcos' como fuerza motriz, en el cuerpo del servidor habita un alma mortal compuesta" (1881, p. 263). Lippert ve en la idea de un alma inmortal y divina el efecto posterior de la religiosidad más antigua y la continuación de una "concepción popular" (1881, p. 263). Este pasaje puede considerarse, por tanto, como una inspiración para la polémica antiplatónica de Nietzsche, según la cual la "superstición del alma" es una "superstición de los pueblos de los tiempos prehistóricos" (KSA 5, p. 11). En tercer lugar, Platón tiene una idea de justicia en la que cada parte del alma tiene y hace lo suyo y, de esta manera, tal como los estoicos, proyecta sus concepciones normativas de un orden justo en la naturaleza (humana) (KSA 5, pp. 21s.). La organización de las partes del alma puede ser para Platón correcta o incorrecta, buena o mala, mientras que para Nietzsche la suposición de una escala de medida externa se ha vuelto inverosímil. La "construcción social de instintos y afectos" expresa una jerarquía de preferencias, pero es categóricamente incorrecto preguntar sobre la naturaleza buena o malvada de este orden. En cuarto lugar se presenta el 
caso de que para Nietzsche los instintos y afectos son de diversas maneras y esencialmente iguales, mientras que para Platón las tres partes limitadas del alma nunca pueden ser del mismo tipo ni contar con el mismo derecho.

En contraste con el especulativo y esencialmente engañoso intento de explicar la antropología de Nietzsche como un refinamiento de lo platónico, la importancia de Der Kampf der Theile im Organismus (La lucha de las partes en el organismo), de Wilhelm Roux (1881), por ejemplo, que es positivamente comprobable, ha sido bien documentada durante más de cuarenta años, y ha sido evidente hasta en la metafórica política (Müller-Lauter, 1978). Roux ve la lucha como un principio regulador a través del cual los elementos en conflicto forman una precaria unidad, que al mismo tiempo se combinan en una transformación dinámica. Además de Roux puede hacerse referencia también a la lectura de Nietzsche realizada por Lange, Espinas, Schneider, Rolph, Drossbach y otros, que también entienden fisiológicamente al organismo como una multiplicidad organizada ${ }^{10}$ : "Todo ser vivo no es un individuo, sino una pluralidad; incluso en la medida en que nos parece un individuo, sigue siendo una unión de seres vivos autónomos", tal como ya ha sido dicho por Goethe (1998 [1817], p. 56) ${ }^{11}$. La multiplicidad necesita organización, pero al mismo tiempo esta organización se basa en una tensión interna continua que la mantiene en desarrollo. Roux precisa que al igual que en un Estado, la diversidad interna y la competencia son necesarias en un organismo, pues él no "se desarrollaría sin esta competencia de los individuos, simplemente a través de la lucha con los Estados vecinos" (Roux, 1881, p. 274). Según Nietzsche, "la batalla de los tejidos se convierte en un principio regulador" (Nachlass 1883 7[190], KSA 10, p. 303).

De acuerdo con esta imagen del sujeto como una organización agonal, Nietzsche hace un apunte en el contexto de su lectura de Roux:

Así como la célula está fisiológicamente junto a la célula, el instinto (Trieb) lo está junto al instinto (Trieb). La imagen más general de nuestra naturaleza es una socialización de instintos (Triebe), con rivalidad constante y alianzas individuales entre sí. El intelecto objeto del concurso (Nachlass 1883 7[94], KSA 10, p. 274).

\footnotetext{
${ }^{10}$ Para conocer acerca de las lecturas de Nietzsche y acerca de su biblioteca personal cfr. Campioni, D'Iorio, Fornari, Fronterotta y Orsucci (2003).

${ }^{11}$ Si Nietzsche no conocía este pasaje directamente de la lectura de Goethe, probablemente lo vio citado en la Historia del materialismo de Friedrich Albert Lange (Lange, 1974 [1873-75], p. 694).
} 
El hombre aparece aquí como una organización sociopolítica de células e instintos (Triebe) que se combinan en acciones individuales, así como en el proceso de vida en su conjunto, para formar una unidad particular. El intelecto no es el soberano controlador de esta competencia, sino el objeto de la misma. Por regla general él sirve a la racionalización y a la explicación apologética de aquello que ha llegado al poder en todo el organismo. Nietzsche considera como un engaño moral la afirmación de que solo el intelecto debe gobernar sobre los otros instintos (Instinkte), porque se estima que solo su voluntad es "libre". "El deseo de superar un afecto es en última instancia, sin embargo, solamente el deseo de otro afecto" (Nachlass 1882/83 5[1.58], KSA 10, p. 194). Esto también explica las críticas de Nietzsche al concepto de voluntad de Schopenhauer. En el sentimiento soberano del volente no se expresa una instancia autosuficiente, sino que "lo que ocurre es que en cada comunidad bien construida y feliz la clase dominante se identifica con los éxitos de la comunidad" (KSA 5, p. 33) ${ }^{12}$. De acuerdo con la jerarquía fisio-política respectiva, uno u otro afecto tiene la supremacía, y este orden se manifiesta en todas las expresiones de vida del sujeto. Cual afecto prevalece es de segundo orden. Para la función y la supervivencia siempre precaria del todo, al principio es crucial que exista una unidad organizada de la voluntad. Si se está buscando un modelo histórico para esta forma de organización política, debe pensarse en Homero en lugar de Platón. La comunidad aristocrática de los aqueos ante Troya se basa en un equilibrio agonista e inestable de obligaciones, expectativas e intereses mutuos (Ulf, 1990). En él se puede ver el siempre precario "antagonismo moderado con poderes aproximadamente iguales" (Siemens, 2016, p. 187), que se identificó de manera convincente como el núcleo de la concepción nietzscheana de la subjetividad soberana. Los aristócratas reunidos participan en la lucha común por razones diferentes. Se organizan y, según la situación, forman una "pluralidad con un

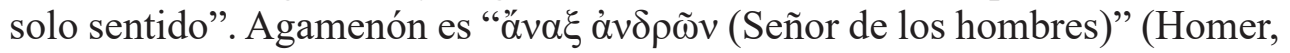
2013, p. 1-442), su voluntad tiene cierta autoridad y su palabra tiene un peso considerable en la congregación. La causa común solo puede tener éxito si, y en la medida, en que logra ganar a los aqueos para ello, ya sea por amenaza o por un incentivo, ya sea por lealtad o cálculo. Al mismo tiempo, el conflicto con Aquiles muestra claramente que ni Agamenón ni una autoridad

\footnotetext{
${ }^{12}$ Lo que se aplica al sujeto según Nietzsche, se aplica a la sociedad según Marx y Engels: "Los pensamientos de la clase dominante son los pensamientos predominantes en cada época, es decir, la clase que es el poder material dominante de la sociedad es al mismo tiempo su poder espiritual dominante" (Marx und Engels, 1986 [1845], p. 46).
} 
superior del deber socio-moral o de los intereses objetivos tienen un simple poder de coerción sobre los otros aristócratas. La discusión de Aquiles con Ulises, Áyax y Fénix, en el noveno libro de la Iliada, ilustra acertadamente que no hay poder externo o interno, ni oferta social o económica, ni argumento político o moral que subordine a Aquiles al interés de la comunidad. Los aristócratas de Troya tienen diferentes fortalezas, habilidades e intereses; entre ellos hay una competencia jerárquica por el prestigio y el honor, aunque todos ellos son del mismo tipo.

Mientras que hasta ahora las dimensiones psicofisiológicas individuales de los hombres en tanto organización corporal estaban en primer plano, en lo subsiguiente el enfoque deberá centrarse en los aspectos intersubjetivos. El individuo humano es biológica y socioculturalmente parte y producto de la historia del género. Si él es entendido en tanto "estructura social de los instintos y los afectos", debe tenerse al mismo tiempo en cuenta que los componentes de esta organización son parte y producto de la historia humana: "Nuestros instintos (Triebe) y pasiones son monstruosas etapas a través de las cuales han sido cultivadas las relaciones sociales y sexuales (a lo mejor previamente en las hordas de simios)" (Nachlass 1881 11[130], KSA 9, p. 487). Nietzsche concibe al sujeto, no solo interna sino también externamente, como una realidad social organizada. Al hacerlo, no solo contradice toda concepción esencialista, sino también la robinsonada especulativa de los conceptos contractualistas: "La sociedad no se configura a partir de individuos ya existentes por medio de un contrato; más bien, es la sociedad la que educa y da forma a los individuos para que sean el producto de la sociedad" (Siemens, 2016, p. 172). En el seno de las asociaciones biológicas, el especimen delimitado físicamente se desarrolla a partir de un órgano, de una función del todo, en una organización más o menos autónoma, que sin embargo permanece constitutivamente relacionada con los demás. Ya hemos visto que Nietzsche considera la construcción de la estabilidad mundial externa como una condición de la subjetividad. Pero las "experiencias egoístas internas" son también de acuerdo con esta imagen "reducidas a nuestras posiciones practicadas y aprendidas en relación con los otros" (Nachlass 1880 6[70], KSA 9, p. 212 ${ }^{13}$. En este sentido, el sujeto está, y sigue estando, indisoluble-

\footnotetext{
${ }^{13}$ Siemens afirma acertadamente: "Incluso en la retirada a la soledad, que a menudo se entiende como sintomática del individualismo autárquico de Nietzsche, practicamos las relaciones sociales con nosotros mismos y todos llevamos nuestros hábitos e impulsos sociales" (Siemens, 2016, p. 181). Por lo tanto, la objeción de que "la defensa del egoísmo de Nietzsche" es una prueba de una forma de pensamiento antisocial tampoco llega a ningún lado.
} 
mente vinculado a la sociedad: "Antaño estuvo el yo oculto en el rebaño: y ahora está el rebaño aun oculto en el yo" (Nachlass 1882 5[1.273], KSA 10, p. 220).

Las aculturadas y encarnadas posiciones respecto a los demás no solo se manifiestan en aquellos instintos (Triebe) referidos a los otros, como por ejemplo el deseo sexual, el amor, la envidia, el odio o la necesidad de reconocimiento, sino también en las 'capacidades superiores' del hombre. La naturaleza social se extiende en particular al lenguaje y, por tanto, a todo aquello que buscamos articular de manera consciente. Esto es particularmente claro en la reinterpretación nietzscheana de la formulación schopenhaueriana del "genio de la especie" (García, 2010). Las expresiones lingüísticas son, por una parte, signos de "grupos de sensaciones" (KSA 5, p. 221), que son esencialmente solipsistas, mientras que, por otro lado, el lenguaje sirve principalmente para el propósito social de la comprensión, especialmente en situaciones de crisis: "Para entenderse uno al otro no es suficiente que se usen las mismas palabras: se deben usar las mismas palabras también para el mismo tipo de experiencia interna, se debe tener, finalmente, una experiencia en común con el otro" (KSA 5, p. 221). La comunidad de experiencia resulta de mundos de la vida y de experiencias compartidas duraderamente a través de las generaciones, y esto permite una rápida comprensión, por ejemplo, al interior del pueblo o entre amigos, parientes y amantes. Sin embargo, el lenguaje solo expresa con éxito las dimensiones de grupos privados de sentimientos, entendibles y comprensibles por parte de otros.

Sin embargo, Nietzsche no solo sostiene aquí que el lenguaje es una institución social y, por tanto, que no es un asunto privado. Más bien, el genio de la especie tiene miedo de "(...) que uno de los dos, usando las mismas palabras, sienta, piense, barrunte, desee, tema de un modo distinto al otro" (2013, p. 289; KSA 5, p. 222). Con esta idea, Nietzsche introduce una conexión entre su concepción fisio-política del sujeto y una socio-fisiología de la especie. Con este fin retoma la idea del sujeto como estructura social, que se desarrolló sobre todo en el primer capítulo de Más allá del bien y del mal: "Cuáles son los grupos de sensaciones que se despiertan más rápidamente dentro de un alma, que toman la palabra, que dan órdenes: eso es lo que decide sobre la jerarquía entera de sus valores (...)", con lo que estas "valoraciones" a su vez dejan reconocer "(...) algo de la estructura de su alma, y nos dicen en qué ve ésta sus condiciones de vida, sus auténticas necesidades” (2013, pp. 289-290; KSA 5, p. 222). Esta concepción está vinculada a un proceso de 
selección que continuamente prefiere lo promedio frente a lo extraordinario. Suponiendo que los hombres con sentimientos similares se entiendan más rápida y más fácilmente, la presión histórica cultural y natural favorece “(...) una fácil comunicabilidad de las necesidades, es decir, en su último fondo, el experimentar vivencias solo ordinarias y vulgares" (Nietzsche, 2013, p. 290; KSA 5, p. 222). Por esta razón sostiene Nietzsche: "Los hombres más similares, más habituales, han tenido y tienen siempre ventaja; los más selectos, más sutiles, más raros, más difíciles de comprender, esos fácilmente permanecen solos en su aislamiento, sucumben a los accidentes y se propagan raras veces" (2013, p. 290; KSA 5, p. 222).

Las realidades sociales y políticas satisfacen siempre las necesidades de la comunidad, en última instancia determinadas por la naturaleza. Ciertamente Nietzsche reconoce esta necesidad e incluso percibe las dimensiones potenciadoras, profundizadoras y refinadoras de la socialización. Sin embargo, su simpatía parece pertenecer claramente a las excepciones y su fracaso produce compasión (KSA 5, pp. 222s.), lo que es comprensible en el contexto de una concepción agonal de transformación. Los inusuales son los heraldos de lo nuevo; el impulso excéntrico en el que se basa el desarrollo. En consecuencia, el aforismo concluye persuasivamente con una invitación: "Es preciso apelar a ingentes fuerzas contrarias para poder oponerse a este natural, demasiado natural, progressus in simil (progreso hacia lo semejante), al avance del hombre hacia lo semejante, habitual, ordinario, gregario - ¡ hacia lo vulgar!-" (Nietzsche, 2013, p. 290; KSA 5, p. 222). Para romper la nivelación se requieren significativos esfuerzos. A la luz de esta conclusión condicional, al final de esta investigación, surgen dos preguntas que revelan el humanismo fundamental de Nietzsche, no en el sentido de una doctrina ideal, sino en el sentido de una filosofía amigable dirigida al hombre real y a sus reales posibilidades: primero, ¿en qué sentido es posible en general impedir el 'progressus' natural? Y, segundo, ¿por qué deberíamos intentarlo?

\section{EI humanismo como una posibilidad humana-sobrehumana}

A diferencia de la tradición dualista de los despreciadores platónico-cristianos del cuerpo, Nietzsche no reconoce la libertad en el dominio supuestamente autosuficiente de una voluntad pura y racional sobre los impulsos conflictivos de la naturaleza interior. Él cuestiona tanto la realidad como la idealidad reguladora de tal libertad. Sin embargo, presupone claramente que es posible y deseable intervenir creativamente en el curso natural de las cosas. 
El hombre, por tanto, parece ser indeterminado en sus acciones y capaz de cambiar el mundo a propósito ${ }^{14}$. Es útil recordar aquí con Ernst Tugendhat la diferencia entre trascendencia ontológica y trascendencia antropológica. De hecho, Nietzsche rechaza la idea de un ser trascendente; en este sentido es un naturalista. Por otro lado, la dimensión antropológica, según la cual los hombres en una actividad del trascender pueden referirse a un espacio de representación más allá de la realidad espacio-temporal, sigue siendo central para él: "Una idea que diferencia a Nietzsche de otros naturalistas es que él, sin embargo, en el trascender humano se adhirió a algo" (Tugendhat, 2007, p. 14). La antropología de Nietzsche está siempre relacionada con una praxis de la superación, a partir de la cual la libertad humana y la trascendencia del mundo interior pueden entenderse de una manera nueva y diferente. Trascender no tiene necesariamente que referirse a un más allá y a un ideal, sino que puede referirse a algo que es posible, a un no-real-aún (Noch-NichtWirkliches). La capacidad para hacerlo resulta del poder transformador y dinámico del propio organismo vivo. Sin embargo, la conveniencia de lo que aún no es real no resulta de su posible valor per se, sino de la fuerza normativa de lo posible. Con esta formulación me refiero a la persuasiva atracción de dar sentido a cualquier posibilidad que haya surgido, a través de su realización intencional en retrospectiva. De esta manera, el superhombre puede ser el significado de la tierra.

Para una mejor comprensión de esta idea, nuevamente es esclarecedora la distinción ante la tradición platónica. En el preludio del Fedro, Sócrates se lamenta: "Hasta ahora y siguiendo la inscripción de Delfos, no he podido conocerme a mí mismo. Me parece ridículo, por tanto, que el que no se conoce todavía, se ponga a pensar lo que ni le va ni le viene" (Platón 1990a, 229e-230a). Según una antigua tradición, a la entrada del templo de Apolo en Delfos estaba escrito: $\Gamma \nu \tilde{\omega} \theta \imath$ $\sigma \varepsilon \alpha v \tau o ́ v$ - conócete a ti mismo. Al poner este requerimiento en el centro de sus esfuerzos intelectuales, Sócrates explica el adecuado conocimiento de uno mismo como condición de toda comprensión consciente. Con la pretensión de autoconocimiento, el individuo se convierte

\footnotetext{
${ }^{14}$ Ocasionalmente Nietzsche ha sido leído como un fatalista-determinista para quien el sujeto está totalmente involucrado en una causalidad natural: "Nietzsche sostiene que la vida de una persona continúa a lo largo de una trayectoria fija, determinada por hechos 'naturales' sobre dicha persona. Nietzsche, el fatalista, ve a una persona como una planta" (Leiter, 2002, p. 81). Esta interpretación expresa una concepción mecanicista de los hechos causales esenciales, que ya no es de Nietzsche. Incluso una mirada más cercana a su concepción de la vida vegetal podría haber aclarado este error, como lo ha demostrado Vanessa Lemm (2016).
} 
simultáneamente en un destinatario crítico del preguntar. Sócrates es desconocido para sí mismo, con los medios del $\lambda o ́ \gamma o \varsigma$ indaga por su esencia supuestamente verdadera. Esta búsqueda se inspira en la esperanza de que podamos encontrar una fecunda respuesta normativa a la pregunta acerca de nosotros mismos. Nietzsche no comparte esta esperanza. En esto difiere también de Volker Gerhardt cuando éste ata la "realidad y efectividad de un concepto íntegro de la individualidad humana", en primer lugar, y ante todo a Sócrates, para luego afirmar: "Solamente con Sócrates se vuelve certeza que un ser humano singular tome conciencia de su singularidad y que en esta conciencia busque la ley de su propia acción" (Gerhard, 1997, p. 12). La pregunta por las leyes de la propia acción no puede resolverse mediante una investigación, especialmente no mediante una búsqueda de razones universalmente vinculantes, sino que debe ser respondida activamente y probada experimentalmente por los hombres y según el criterio humano.

Por esta razón Nietzsche no suspende de ninguna manera la pregunta por el sí-mismo, sino que determina el objeto del conocimiento, los instrumentos del autoconocimiento, así como el objetivo de los esfuerzos, de una manera fundamentalmente diferente a la del Sócrates platónico. No comprende el sí-mismo como una entidad espiritual estable, ni cree que éste pueda ser captado adecuadamente con los instrumentos del análisis teórico. En el aforismo de La gaya ciencia “¡Arriba la física!” resalta: “"Cada uno es para sí mismo el más lejano» -eso lo saben, para su desasosiego, todos los que ponen a prueba los riñones de los hombres; y la sentencia «¡conócete a ti mismo!» es, en boca de un Dios y dicho a los hombres, casi una maledicencia" (Nietzsche, 1990, p. 192; KSA 3, p. 560). Los hombres están limitados en este esfuerzo; el gran riesgo, especialmente, es confundir el autoconocimiento con la construcción egoísta de una autoimagen moralmente sólida. En lugar de la introspección mojigata, Nietzsche se basa en la "purificación de nuestras opiniones y valoraciones" y, luego, sobre todo, en la "(...) creación de nuestras propias nuevas tablas de lo que es bueno" (1990, p. 194; KSA 3, p. 563).

El elogio de la física (de la fisiología, del naturalismo) y el conocimiento de "todo lo que es legal y necesario en el mundo" se limita a su función útil para este propósito: "tenemos que ser físicos, para poder ser creadores en ese sentido" (1990, p. 195; KSA 3, p. 563). Frente a los intentos idealistas de diseñar nuevos valores sin tener en cuenta la física, habla finalmente lo que "nosotros" valoramos aún más que la física y "(...) lo que nos obliga a ir hacia ella: ¡nuestra honestidad!” (1990, p. 195; KSA 3, p. 564). Probablemente ha sido también esta honestidad la que muestra los límites del conocimiento 
y de las acciones humanas. Es por esto que Nietzsche pone en el lugar del 'nosce te ipsum' otra demanda: "Sin embargo, nosotros queremos llegar a ser los que somos - ¡los nuevos, los únicos, los incomparables, los que-sedan-leyes-a-sí-mismos, los que-se-crean-a-sí-mismos!” (1990, pp. 194-195; KSA 3, p. 563). La pregunta 'quién se es' ya no es un problema epistémico en el contexto de la antropología fisio-política aquí expuesta. Ya no se trata de descubrir y sacar a la luz la naturaleza oculta de los seres humanos a través de la introspección, el análisis, la neurociencia u otras formas de investigación metódica. 'Quién se es' no es una cuestión teórica ni tampoco ética, sino práctica.

Una idea más precisa de esta concepción creativa y radicalmente autónoma del hombre es transmitida por otro aforismo de La gaya ciencia:

Una cosa es necesaria «Dar estilo» al propio carácter - $i$ un arte grande y escaso! Lo ejerce aquel cuya vista abarca todo lo que de fuerzas y debilidades le ofrece su naturaleza, y luego les adapta un plan artístico hasta que cada una aparece como arte y razón, en donde incluso la debilidad encanta al ojo. Aquí se agregó una gran masa de naturaleza de segunda, allá se quitó un trozo de naturaleza de primera -en ambas ocasiones, luego de un largo ejercicio y trabajo diario con ello (1990, p. 167; KSA 3, p. 530).

El carácter pleno de estilo consiste en la combinación de formación cultural y dispositivos naturales para una obra de arte de buen gusto (Heit, 2016b). Para esto se necesita en primer lugar una visión general de las diversas capacidades del organismo, un conocimiento de los instintos (Triebe) y afectos. Pero también se requiere de un concepto estético, de un plan y de una voluntad que unan las diferentes partes en una organización. Finalmente se requiere, en tercer lugar, la paciente praxis, ya que la jerarquía de los instintos (Triebe) y los afectos solamente se deja desplazar con dificultad. La autoconfiguración se lleva a cabo también en el ejercicio práctico de los pequeños detalles, como en la respiración, la morada, el descanso, la nutrición física y mental. En una personalidad exitosa “(...) se revela que era la coacción del mismo gusto la que dominaba y daba forma a lo grande y a lo pequeño: poco importa si era un buen o un mal gusto, si se piensa que -ibasta con que sea un gusto!" (1990, p. 167; KSA 3, p. 530). En contraste con la "semejanza con el Dios" platónico-moral (Platón 1990b, 176b), el objetivo de esta autoconfiguración ya no es religioso (imitatio Christi) o cuasirreligioso (armonía con la naturaleza), moral (virtud, deber) o social (honor, reconocimiento, prestigio), sino explícitamente estético. 
Es posible que de esta manera los hombres crezcan más allá de sí mismos. Con Nietzsche la vida puede ser entendida como un proceso continuo de organización fisio-política y de autorebasamiento. Mientras algo está vivo, se forma, se organiza y se transforma; en el proceso se constituye como realidad, y al mismo tiempo se proyecta siempre más allá de la realidad presente. Como funciones socialmente necesarias, el animal humano dispone de memoria, lenguaje, voluntad, conciencia, pequeña y gran razón. Estas funciones son de origen histórico de la especie y son precarias en su respectiva efectividad. Precisamente la pequeña razón es sobreestimada fácilmente. Sin embargo, ellas son reales como productos de la naturaleza humana y de la historia cultural. Las posibilidades y habilidades surgidas de esta manera abren al hombre, y a los hombres, nuevas posibilidades para una bella configuración. Mientras que el dualismo deriva en la capacidad para la libertad del dominio autónomo de la razón sobre los peligrosos impulsos de la naturaleza interna y externa, el naturalismo mecánico niega completamente toda posibilidad de una creativa autoconfiguración. Nietzsche evita esta falsa alternativa y entiende la libertad como precaria capacidad de una organización fisio-política. El tipo de organización y, con ello, también la responsabilidad por sus consecuencias, reside en nosotros. Con el acabamiento de la autarquía substancial y de la universalidad de la razón, los ideales del humanismo ya no pueden reclamar una validez universal. Ellos son solamente representaciones humanas-demasiado humanas, que se deben a una historia no extrañamente violenta. Pero en la medida en que esta historia abra la posibilidad de un embellecimiento del hombre, Nietzsche y Gerhardt, a lo mejor, están nuevamente de acuerdo en el punto central, en aquel punto en que este último exige que el hombre tenga "en la conciencia de su responsabilidad con su mundo (...) el coraje de ser un hombre hoy" (Gerhardt, 2019, p. 13). Esa es quizás la tarea ultrahumana.

\section{Referencias}

Abel, G. (2001). Bewußtsein - Sprache - Natur: Nietzsches Philosophie des Geistes. Nietzsche-Studien, 30, pp. 1-43.

Campioni, G., D’Iorio, P., Fornari, M. C., Fronterotta, F., Orsucci, A. (2003). Nietzsches persönliche Bibliothek. (Colaboración de Renate MüllerBuck). Berlin / New York: Walter de Gruyter. 
Clark, M. and Dudrick, D. (2012). The Soul of Nietzsche's Beyond Good and Evil. Cambridge: Cambridge University Press.

Clark, M. and Dudrick, D. (2015). Nietzsche's Philosophical Psychology: Will to Power as Theory of the Soul. Clark, M (Hrsg.). Nietzsche on Ethics and Politics (pp. 260-286). Oxford: Oxford University Press.

Gerhardt, V. (1997) Das individuelle Gesetz. Über eine sokratisch-platonische Bedingung der Ethik. Allgemeine Zeitschrift für Philosophie, $22(1)$, pp 3-21.

Gerhardt, V. (2011). Die „grosse Vernunft“ des Leibes. Ein Versuch über Zarathustras vierte Rede. Heilinger, J. Ch. und Loukidelis, N (Hrsg.). Die Funken des freien Geistes. Neuere Aufsätze zu Nietzsches Philosophie der Zukunft. (pp. 50-86). Berlin: Walter de Gruyter.

Gerhardt, V. (2019). Humanität. Über den Geist der Menschheit. München: C. H. Beck.

Goethe, J. W. von (1998 [1817]). „Zur Morphologie“. In: Blumenthal, Lieselotte et al. (Hrsg.). Johann Wolfgang von Goethe Werke (B. 13). München: dtv.

Heit, H. (2013). „... Was man ist“"? Zur Wirklichkeit des Subjekts bei Nietzsche. (R. Reschke Hrsg.). Nietzscheforschung 20 (pp. 173-192). Berlin: Akademie Verlag.

Heit, H. (2016a): Naturalizing Perspectives. On the Epistemology of Nietzsche's Experimental Naturalizations. Nietzsche-Studien, 45, pp. 56-80.

Heit, H. (2016b). Stilvolles Durchwursteln. Nietzsche und die Lebenskunst im Zeitalter der Beschleunigung. Gödde, G. Loukidelis, N und Zirfas, J (Eds.) Nietzsche und die Lebenskunst. Ein philosophisch-psychologisches Kompendium (pp. 307-315). Stuttgart: J. B. Metzler.

Heit, H (2018). Der Mensch als „Gesellschaftsbau“. Nietzsches PhysioPolitische Anthropologie der Freiheit. Internationales Jahrbuch für Philosophische Anthropologie, 7, pp. 101-118.

Helmholtz, H. von (1867). Handbuch der physiologischen Optik. Leipzig: Leopold Vo. 
Homer (2013). Ilias (Griechisch-Deutsch). Herausgabe und Übersetzung von Hans Rupé. Berlin: Walter de Gruyter.

Ioan, R. (2015). Philosophical Physiology: Schopenhauer and Nietzsche. Ribeiro dos Santos, L and Dawn Hay, K (Hrsg.). Nietzsche, German Idealism and Its Critics (pp. 208-222). Berlin: Walter de Gruyter.

Janaway, Ch. (2014). Nietzsche's Psychology as a Refinement of Plato's. The Journal of Nietzsche Studies, 45 (1), pp. 12-21.

Kim, J. H. (1995). Nietzsches Sozialphilosophie. Versuch einer Überwindung der Moderne im Mittelpunkt des Begriffes „Leib“. Würzburg: Königshausen \& Neumann.

Lange, F. (1974 [1873/75]. Die Geschichte des Materialismus und Kritik seiner Bedeutung in der Gegenwart. Frankfurt a. M.: Suhrkamp.

Leiter, B. (2002). Nietzsche on Morality. London: Routledge.

Lemm, V. (2016): Is Nietzsche a Naturalist?: Or How to Become a Responsible Plant. The Journal of Nietzsche Studies, 47 (1), pp. 61-80.

Lippert, J. (1881). Die Religionen der europäischen Culturvölker: der Litauer, Slaven, Germanen, Griechen und Römer, in ihrem geschichtlichen Ursprunge. Berlin: Hofmann.

Marx, K. und Engels, F. (1986 [1845]). Die deutsche Ideologie. Institut für Marxismus Leninismus beim ZK der SED (Hrsg.). Karl Marx Friedrich Engels - Werke (MEW) (pp. 9-530). Berlin: Dietz.

Muniz Garcia, A. (2010). Nietzsches Umdeutung von Schopenhauers "Genius der Gattung» in Jenseits von Gut und Böse 268 und Fröhliche Wissenschaft 354. Campioni, G. Piazzesi, Ch. Wotling, e P. (Hrsg.). Letture della Gaia scienza. Lectures du Gai savoir (pp. 143-156). Pisa: Edizioni ETS.

Müller-Lauter, W. (1978). Der Organismus als innerer Kampf. Der Einfluss von Wilhelm Roux auf Friedrich Nietzsche. Nietzsche-Studien, 7, pp. 189-235.

Nietzsche, F. (1988). Die Geburt der Tragödie. Unzeitgemäße Betrachtungen. Nachgelassene Schriften 1870-1873 (KSA 1). Kritische Studien Ausgabe. Herausgegeben von Giorgio Colli und Mazzino Montinari. Berlin: Walter de Gruyter. 
Nietzsche, F. (1988). Morgenröte. Idyllen aus Messina. Die fröhliche Wissenschaft (KSA 3). Kritische Studien Ausgabe. Herausgegeben von Giorgio Colli und Mazzino Montinari. Berlin: Walter de Gruyter.

Nietzsche, F. (1988). Also sprach Zarathustra I-IV(KSA4). Kritische Studien Ausgabe. Herausgegeben von Giorgio Colli und Mazzino Montinari. Berlin: Walter de Gruyter.

Nietzsche, F. (1988). Jenseits von Gut und Böse. Zur Genealogie der Moral (KSA 5). Kritische Studien Ausgabe. Herausgegeben von Giorgio Colli und Mazzino Montinari. Berlin: Walter de Gruyter.

Nietzsche, F. (1988). Nachgelassene Fragmente 1880-1882 (KSA 9). Kritische Studien Ausgabe. Herausgegeben von Giorgio Colli und Mazzino Montinari. Berlin: Walter de Gruyter.

Nietzsche, F. (1988). Nachgelassene Fragmente 1882-1884 (KSA 10). Kritische Studien Ausgabe. Herausgegeben von Giorgio Colli und Mazzino Montinari. Berlin: Walter de Gruyter.

Nietzsche, F. (1988). Nachgelassene Fragmente (KSA 11). Kritische Studien Ausgabe. Herausgegeben von Giorgio Colli und Mazzino Montinari. Berlin: Walter de Gruyter.

Nietzsche, F. (1990). La ciencia jovial. (Trad. Jara, J). Caracas: Monte Ávila editores.

Nietzsche, F. (1993). Así habló Zaratustra. (Trad. Sánchez Pascual, A). Madrid: Alianza.

Nietzsche, F. (2013). Más allá del bien y del mal. (Trad. Sánchez Pascual, A). Madrid: Alianza.

Platón. (1990a). Phaidros. Werke (5). Günter, E (Hrsg.). Darmstadt: WBG Verlag.

Platón. (1990b). Theaitetos. Werke (6). Günter, E (Hrsg.). Darmstadt: WBG Verlag.

Roux, W. (1881). Der Kampf der Theile im Organismus. Ein Beitrag zur Vervollständigung der mechanischen Zweckmässigkeitslehre. Leipzig: Engelmann. 
Schacht, R. (2012). Nietzsche's Anti-Scientistic Naturalism. Heit, H. Abel, $\mathrm{G}$ und Brusotti, M (Hrsg.). Nietzsches Wissenschaftsphilosophie (pp. 161-186). Berlin: Walter de Gruyter.

Schiemann, G. (2014). Nietzsche und die Wahrheitsgewissheitsverluste im Anbruch der Moderne. Heit, H. und Heller, L (Hrsg.). Handbuch Nietzsche und die Wissenschaften. Natur-, geistes- und sozialwissenschaftliche Kontexte (pp. 46-75). Berlin: Walter de Gruyter.

Siemens, H. (2016). Nietzsches Sozio-Physiologie des Selbst und das Problem der Souveräntität. Heit, H. und Thorgeirsdottir, S (Hrsg.). Nietzsche als Kritiker und Denker der Transformation (pp. 167-188). Berlin: Walter de Gruyter.

Sommer, A. (2016). Kommentar zu Nietzsches Jenseits von Gut und Böse. Berlin: Walter de Gruyter.

Tugendhat, E. (2007). Anthropologie statt Metaphysik. München: C. H. Beck.

Ulf, Ch. (1990). Die homerische Gesellschaft. Materialien zur analytischen Beschreibung und historischen Lokalisierung. München: C. H. Beck. 